\title{
Characteristics-based Boundary Conditions for the Euler Adjoint Problem: 2-D Formulation
}

\author{
Marcelo Hayashi* \\ University of São Paulo, São Paulo, SP, 05508-970, Brazil \\ Marco Ceze ${ }^{\dagger}$ \\ University of Michigan, Ann Arbor, MI, 48105, United States \\ Ernani Volpe $\mathrm{f}^{\ddagger}$ \\ University of São Paulo, São Paulo, SP, 05508-970, Brazil
}

\begin{abstract}
Over the last decade the adjoint method has been consolidated as one of the most versatile and successful tools for aerodynamic design. It has become a research area on its own, spawning a large variety of applications and a prolific literature. Yet, some relevant aspects of the method remain relatively less explored in the literature. Such is the case with the adjoint boundary conditions and, more specifically, with regard to permeable boundaries. The present work discusses at length a novel approach to the continuous adjoint boundary problem, with emphasis on the full characteristic formulation of the farfield boundary conditions. The main goal of this approach is to ensure the well-posedness of the adjoint equations and consistency with the primal problem.
\end{abstract}

\section{Introduction}

The problem of inverse aerodynamic design was first considered by Lighthill, in 1945, and the investigation was limited to airfoils in incompressible potential flows. ${ }^{1}$ Over the years, a variety of methods has been proposed to tackle the problems of aerodynamic optimization and inverse design. Eventually, with the advances in aerospace sciences, the compressible flow regime has also been addressed, starting with such classical works as those by Hicks et. al., Garabedian-MacFadden, ${ }^{2-4}$ and followed by many others.

The adjoint method has played a prominent role in that context, for a number of reasons. Among them one could cite the great flexibility it offers with regard to the flow-physics model and to the definition of objective functionals. Originally proposed by Pirronneau ${ }^{5-7}$ for elliptic problems, it was later extended to transonic flows by Jameson. ${ }^{1}$ Since then, it has become the subject of extensive research activity, ${ }^{8-17}$ and spawned a wide variety of applications, ranging from nuclear reactor thermo-hydraulics to atmospheric sciences. ${ }^{18,19}$

In aerodynamics, the developments of the adjoint method encompass design applications regarding internal and external flows ${ }^{20-25}$ and, more recently, unsteady flows. ${ }^{26-29}$ An entirely different area of research has evolved around the ideas of error analysis ${ }^{30,31}$ and grid adaptation. ${ }^{31-34}$ It makes use of the adjoint variables to improve the accuracy of functionals, which measure desired qualities of the flow solution. ${ }^{35-38}$

To put matters into perspective, objective functionals of general interest in aerodynamics depend on flow variables and on the shape and location of the boundaries. ${ }^{39,40}$ These, in turn, are controlled by a set of design parameters. For all practical purposes the set is assumed to be finite. Under these circumstances, a natural means of estimating the sensitivity of that functional, to changes in flow geometry, would be to perturb each design parameter individually, and then to compute the sensitivity gradient by finite differences.

The procedure clearly requires a converged flow solution for each parameter variation. As the number of design parameters increases, so does the the number of solutions, and the computational cost is bound

\footnotetext{
*Graduate Student, Department of Mechanical Engineering. Email: mhayashi@usp.br

$\dagger$ Graduate Student, Department of Aerospace Engineering, AIAA Member. Email: mceze@umich.edu

$\ddagger$ Assitant Professor, Department of Mechanical Engineering. Email: ernvolpe@usp.br
} 
to become prohibitive. Alternatively, by imposing the equations that govern the flow as constraints to the optimization problem, one precludes unrealizable solutions. That, in turn, eliminates the need for additional flow simulations. Therein lies the essence of the adjoint method. As a result of the simplification, the method offers an extremely attractive capability, which is to compute sensitivity gradients at a cost that is largely independent of the number of design parameters.

The formulation of the adjoint problem has evolved into two distinct approaches: discrete and continuous. Comparisons between the two approaches have also been drawn in the literature, ${ }^{40-42}$ and they do not seem to indicate any sizable differences between them, in terms of optimization effectiveness.

Yet, despite all the research that has been carried out on the method, it appears that some of its more conceptual aspects have not received the same attention. Such seems to be the case with the mathematical properties of the adjoint solution and, to a greater extent, with its boundary problem. In the literature, these topics have been addressed in a series of seminal articles by Giles and Pierce. ${ }^{43-46}$ However their analysis of the boundary problem is, in a sense, limited to the quasi-1D Euler flow.

The adjoint contour problem is far more involved in multi-dimensional flows, given the variety of boundary conditions and geometry they can incur. Although homogeneous conditions may suffice at the far-field boundary in many applications of external aerodynamics, the same cannot hold for internal flows. Besides, there still remains the relevant question as to under which conditions the adjoint problem is well-posed.

The continuous formulation of the method offers the possibility of a careful investigation into the topic. In view of the similarity the flow and the corresponding adjoint equations exhibit in this framework, it seems reasonable to consider the conditions for well-posedness of the latter on the basis of those that hold for the former. That rationale has been pursued at length in a recent paper by one of us. ${ }^{47}$ However the analysis focused on the quasi-1D Euler flow, so that the results could be verified by comparison with the analytical solutions by Giles and Pierce. ${ }^{46}$

The purpose of this paper is to extend that analysis to multi-dimensional flows. For the sake of clarity and space, we pick the 2-D compressible Euler flow as a suitable vehicle to present the concepts.

\section{The variational problem}

Objective functionals of interest in aerodynamics depend on the flow variables and on the geometry of the boundaries. In principle, these functionals could either be volume integrals over the flow domain, or surface integrals over a boundary such as the body surface, for instance. The latter case is of special relevance to us. For it includes a variety of aerodynamic measures of merit, ranging from force coefficients to inverse design. A fairly general representation of these functionals would be:

$$
I_{o}=\int_{B_{w}} g(\mathbf{V}) \mathrm{d} S
$$

where $B_{w}$ stands for the body surface, a function of space coordinates that is fully specified by a finite set of parameters $B_{w} \Rightarrow S\left(\xi^{j} ; a^{k}\right)$. The quantity $g(\mathbf{V})$ represents a scalar function of the flow variables $\mathbf{V}=\left(\rho, u^{i}, p\right)^{T}$ — which can naturally be put in terms of the conservative variables $\mathbf{Q}=\left(\rho, \rho u^{i}, e\right)^{T}$, as well.

A relevant problem would be to find out a surface geometry that leads to a minimum of $I_{o}$, under given flow conditions. In the framework of the adjoint method, it implies evaluating the sensitivity of $I_{o}$ to changes in the control parameters $a^{k}$. To first order, that can be estimated by the sensitivity gradient: $\partial I_{o} / \partial a^{k}$.

Parameter variations clearly cause the body surface to change, thereby perturbing the flow field as well, $\delta a^{k} \Rightarrow \delta S \Rightarrow \delta \mathbf{Q}$. A convenient means of separating physical from geometric variations is to define a spatial transformation, whereby the body surface is mapped onto a constant coordinate plane. It also simplifies the variational problem considerably, in that the domains of integration remain fixed in transformed space. ${ }^{48}$ All that change in the process are the transformation operators and the metrics of the latter.

To that end, we take the physical space to be represented by Cartesian coordinates $x^{i^{\prime}}$, and the transformed space by generalized coordinates $\xi^{k}$ — to distinguish between the two systems, all tensor elements in Cartesian coordinates will hereafter be designated by primed indices, unprimed indices are reserved for generalized elements. The transformation operator and its inverse are defined by, respectively:

$$
\beta \Rightarrow \beta_{j}^{i^{\prime}}=\frac{\partial x^{i^{\prime}}}{\partial \xi^{j}} \quad ; \quad \beta^{-1} \Rightarrow \beta_{i^{\prime}}^{j}=\frac{\partial \xi^{j}}{\partial x^{i^{\prime}}}
$$


The transformation Jacobian is $J=\operatorname{det}\left(\beta_{j}^{i^{\prime}}\right)$, and it is assumed to be independent of time. It is also understood that $J$ should not go to zero nor change its sign anywhere in $\mathcal{D}$. In this context, the first variation of the objective functional (1) is given by:

$$
\delta I_{o}=\int_{B_{w}} \frac{\partial g}{\partial \mathbf{V}} \frac{\partial \mathbf{V}}{\partial \mathbf{Q}} \delta \mathbf{Q}\left|\frac{\mathrm{d} S^{\prime}}{\mathrm{d} S}\right| \mathrm{d} S+\int_{B_{w}} g \delta\left|\frac{\mathrm{d} S^{\prime}}{\mathrm{d} S}\right| \mathrm{d} S
$$

where $\mathrm{d} S^{\prime}$ and $\mathrm{d} S$ represent area elements in Cartesian and generalized coordinates, respectively. The above eq. (3) naturally involves physical and geometric variations. The latter term can usually be derived from $S\left(\xi^{i} ; a_{k}\right)$, analytically, whereas the former is hardly ever known in closed form. In effect, it is the presence of that term in $\delta I_{o}$ that gives rise to the need for additional flow simulations.

In order to circumvent such need, one imposes the fluid mechanics equations as constraints on the variational problem. They play the essential role of realizability conditions, which all physical variations $\delta \mathbf{Q}$ must satisfy. In the present case, we pick the Euler equations, which are given in Cartesian coordinates by:

$$
\frac{\partial Q_{\alpha}}{\partial t}+\frac{\partial f_{\alpha}^{k^{\prime}}}{\partial x^{k^{\prime}}}=0
$$

The state $Q_{\alpha}$ and flux $f_{\alpha}^{k^{\prime}}$ vectors are defined as

$$
Q_{\alpha} \Rightarrow\left(\frac{\rho}{\rho u^{i^{\prime}}} \frac{;}{e}\right) \quad f_{\alpha}^{k^{\prime}} \Rightarrow\left(\frac{\rho u^{k^{\prime}}}{\frac{\rho u^{i^{\prime}} u^{k^{\prime}}+p g^{i^{\prime} k^{\prime}}}{(e+p) u^{k^{\prime}}}}\right)
$$

The symbol $e$ represents total energy, $e=\rho\left(e_{i}+\mathbf{u} \cdot \mathbf{u} / 2\right), e_{i}$ denotes the specific internal energy. The set is closed by the ideal gas relation between pressure and internal energy

$$
p=\rho e_{i}(\gamma-1)
$$

The Euler equations in transformed space are obtained by a simple application of the $\beta$ operators: ${ }^{49}$

$$
\begin{aligned}
J \frac{\partial Q_{\alpha}}{\partial t}+J \beta_{i^{\prime}}^{k} \frac{\partial f_{\alpha}^{i^{\prime}}}{\partial \xi^{k}} & =0 \\
\frac{\partial\left(J Q_{\alpha}\right)}{\partial t}+\frac{\partial F_{\alpha}^{k}}{\partial \xi^{k}} & =0
\end{aligned}
$$

A tensorial identity ${ }^{50}$ is crucial to derive their latter form, and it is also implied in the definition of generalized flux vectors $F_{\alpha}^{k}$,

$$
\frac{\partial\left(J \beta_{i^{\prime}}^{k}\right)}{\partial \xi^{k}}=0 \quad \Rightarrow \quad F_{\alpha}^{k}=J \beta_{i^{\prime}}^{k} f_{\alpha}^{i^{\prime}}
$$

Generalized flux-Jacobian matrices are then defined on the basis of the above eq. (8)

$$
C_{\alpha \beta}^{k}=J \beta_{i^{\prime}}^{k} \frac{\partial f_{\alpha}^{i^{\prime}}}{\partial Q_{\beta}}=J \beta_{i^{\prime}}^{k} A_{\alpha \beta}^{i^{\prime}}
$$

and they lead to the following form of the generalized Euler equations

$$
\frac{\partial Q_{\alpha}}{\partial t}+\frac{C_{\alpha \beta}^{k}}{J} \frac{\partial Q_{\beta}}{\partial \xi^{k}}=0
$$

In the applications that are considered here, one is mostly interested in steady flow conditions. Therefore, the steady form of (7) is imposed on (1) as a non-holonomic constraint, in transformed space. The procedure leads to an augmented functional, which is given by

$$
I=\underbrace{\int_{B_{w}} g(\mathbf{V})\left|\frac{\mathrm{d} S^{\prime}}{\mathrm{d} S}\right| \mathrm{d} S}_{I_{o}}+\underbrace{\int_{\mathcal{D}} \Psi_{\alpha} \frac{\partial F_{\alpha}^{k}}{\partial \xi^{k}} \mathrm{~d} \mathcal{V}}_{I_{c}}
$$

3 of 18 
where $I_{c}$ indicates the constraint functional, which is a volume integral over the flow domain $\mathcal{D}$. The ratio between Cartesian and generalized volume elements is given by transformation Jacobian itself, $\mathrm{d} \mathcal{V}^{\prime} / \mathrm{d} \mathcal{V}=J$. The vector $\Psi_{\alpha}$ represents Lagrange multipliers and it is of the same dimension as the state vector $Q_{\alpha}$.

The first variation of $I_{c}$ involves the variation of the constraint equation, which is given by

$$
\frac{\partial}{\partial \xi^{k}}\left(\delta F_{\alpha}^{k}\right)=0
$$

where the generalized flux variation is obtained by combining eqs. (8) and (9), the procedure yields

$$
\delta F_{\alpha}^{k}=C_{\alpha \beta}^{k} \delta Q_{\beta}+\delta\left(J \beta_{i^{\prime}}^{k}\right) f_{\alpha}^{i^{\prime}}
$$

It is worth noting that the first term on the RHS of (13) can be regarded as the physical part of the flux variation, whereas the second one represents the geometric part.

The first variation of $I_{c}$ is a direct result of eq. (12), and it can be further simplified by making use of Gauss' theorem.

$$
\delta I_{c}=\int_{\partial \mathcal{D}} \Psi_{\alpha} \delta F_{\alpha}^{k} n_{k} \mathrm{~d} S-\int_{\mathcal{D}} \delta F_{\alpha}^{k} \frac{1}{J} \frac{\partial\left(J \Psi_{\alpha}\right)}{\partial \xi^{k}} \mathrm{~d} \mathcal{V}
$$

where the symbol $\partial \mathcal{D}$ indicates the whole boundary of the flow domain, which involves the body surface $\left(B_{w}\right)$, the far-field $\left(B_{\infty}\right)$ and cut-planes $\left(B_{c p}\right)$. The term $n_{k}$ represents a normal unit vector that points outward from the flow domain.

The variation of the augmented functional (11) is given by the sum of eqs. (3) and (14): $\delta I=\delta I_{o}+\delta I_{c}$. On adding them up and on separating the surface integrals according to each boundary, one gets:

$$
\begin{aligned}
\delta I & =\int_{B_{w}} \frac{\partial g}{\partial Q} \delta Q\left|\frac{\mathrm{d} S^{\prime}}{\mathrm{d} S}\right| \mathrm{d} S+\int_{B_{w}} g \delta\left|\frac{\mathrm{d} S^{\prime}}{\mathrm{d} S}\right| \mathrm{d} S-\int_{\mathcal{D}} \delta F_{\alpha}^{k} \frac{1}{J} \frac{\partial\left(J \Psi_{\alpha}\right)}{\partial \xi^{k}} \mathrm{~d} \mathcal{V}+ \\
& +\int_{B_{w}} \Psi_{\alpha} \delta F_{\alpha}^{k} n_{k} \mathrm{~d} S+\int_{B_{\infty}} \Psi_{\alpha} \delta F_{\alpha}^{k} n_{k} \mathrm{~d} S+\left[\int_{B_{c p}} \Psi_{\alpha} \delta F_{\alpha}^{k} n_{k} \mathrm{~d} S\right]_{c p^{+}}^{c p^{-}}
\end{aligned}
$$

Here, the square brackets imply that the flux variations are integrated over both sides of each cut plane $B_{c p}$. Owing to the fact that the normal has opposite orientation on each side, any cut-plane yields a difference between two similar integrals, one over each side. Moreover, the fluxes should be continuous through the cut planes to be physically realizable, and the same must hold for their variations. Under these circumstances, one can eliminate that term from eq. (15), by simply imposing periodic boundary conditions on the adjoint variables at the cut planes,

$$
\left.\Psi_{\alpha}\right|_{c p^{+}}=\left.\Psi_{\alpha}\right|_{c p^{-}}
$$

It requires that the $\Psi_{\alpha}$ and all of their derivatives be continuous through $B_{c p}$. Another condition for physical realizability implies that the normal velocity at the solid walls should be zero, $\mathbf{u} \cdot \mathbf{n}=0$. That requirement constrains the flux vector normal to $B_{w}$, and it should also hold for its first variation.

$$
\left.F_{\alpha}^{k} n_{k}\right|_{B_{w}}=\left.\left(\begin{array}{c}
0 \\
p J \beta_{i^{\prime}}^{k} g^{i^{\prime} j^{\prime}} n_{k} \\
0
\end{array}\right) \Rightarrow \delta F_{\alpha}^{k} n_{k}\right|_{B_{w}}=\left(\begin{array}{c}
0 \\
\delta p J \beta_{i^{\prime}}^{k} g^{i^{\prime} j^{\prime}} n_{k} \\
0
\end{array}\right)+\left(\begin{array}{c}
0 \\
p \delta\left(J \beta_{i^{\prime}}^{k}\right) g^{i^{\prime} j^{\prime}} n_{k} \\
0
\end{array}\right)
$$

and on assuming that wall is mapped onto a constant coordinate plane, say $B_{w} \Rightarrow \xi^{2}=0$, one can write

$$
\left.\Psi_{\alpha} \delta F_{\alpha}^{k} n_{k}\right|_{\xi^{2}=0}=\delta p\left[\Psi_{\left(i^{\prime}+1\right)} J \beta_{i^{\prime}}^{2} n_{2}\right]+p\left[\Psi_{\left(i^{\prime}+1\right)} \delta\left(J \beta_{i^{\prime}}^{2}\right) n_{2}\right]
$$

it is implied that the metric tensor coincides with the identity matrix in the Cartesian system. 
On substituting eqs. (16) and (18) for the corresponding terms in eq. (15), and on replacing the flux variation by eq. (13), one obtains the final expression for $\delta I$

$$
\begin{aligned}
\delta I= & \underbrace{\int_{B_{w}}\left\{\frac{\partial g}{\partial Q_{\beta}}\left|\frac{\mathrm{d} S^{\prime}}{\mathrm{d} S}\right|+\left[\Psi_{\left(i^{\prime}+1\right)} J \beta_{i^{\prime}}^{2} n_{2}\right] \frac{\partial p}{\partial Q_{\beta}}\right\} \delta Q_{\beta} \mathrm{d} S}_{(\mathrm{a})}+\underbrace{\int_{B_{\infty}} \Psi_{\alpha} C_{\alpha \beta}^{2} \delta Q_{\beta} n_{2} \mathrm{~d} S}_{(\mathrm{b})}-\underbrace{\int_{\mathcal{D}} \frac{C_{\alpha \beta}^{k}}{J} \frac{\partial\left(J \Psi_{\alpha}\right)}{\partial \xi^{k}} \delta Q_{\beta} \mathrm{d} \mathcal{V}}_{(\mathrm{c})} \\
& +\underbrace{\int_{B_{w}}\left\{g \delta\left|\frac{\mathrm{d} S^{\prime}}{\mathrm{d} S}\right|+p\left[\Psi_{\left(i^{\prime}+1\right)} \delta\left(J \beta_{i^{\prime}}^{2}\right) n_{2}\right]\right\} \mathrm{d} S}_{(\mathrm{d})}+\underbrace{\int_{B_{\infty}} \Psi_{\alpha} \delta\left(J \beta_{i^{\prime}}^{2}\right) f_{\alpha}^{i^{\prime}} n_{2} \mathrm{~d} S}_{(\mathrm{e})}-\underbrace{\int \frac{\delta\left(J \beta_{i^{\prime}}^{k}\right) f_{\alpha}^{i^{\prime}}}{J} \frac{\left(J \Psi_{\alpha}\right)}{\partial \xi^{k}} \mathrm{~d} \mathcal{V}}_{(\mathrm{f})}
\end{aligned}
$$

Here it has been assumed that the far-field boundary is also mapped onto a constant coordinate plane, $B_{\infty} \Rightarrow \xi^{2}=1$. The first three terms, $a, b$ and $c$ give rise to the adjoint problem, which is constructed with the specific purpose of eliminating physical variations from the total, $\delta I$. The remaining three integrals, $d, e$ and $f$, involve only geometric variations, and they can be carried over into the sensitivity gradient as they are $^{51}$ However, Jameson and Sangho ${ }^{50}$ have proposed a further simplification to the gradient expression. They have shown that one can use the reduced form:

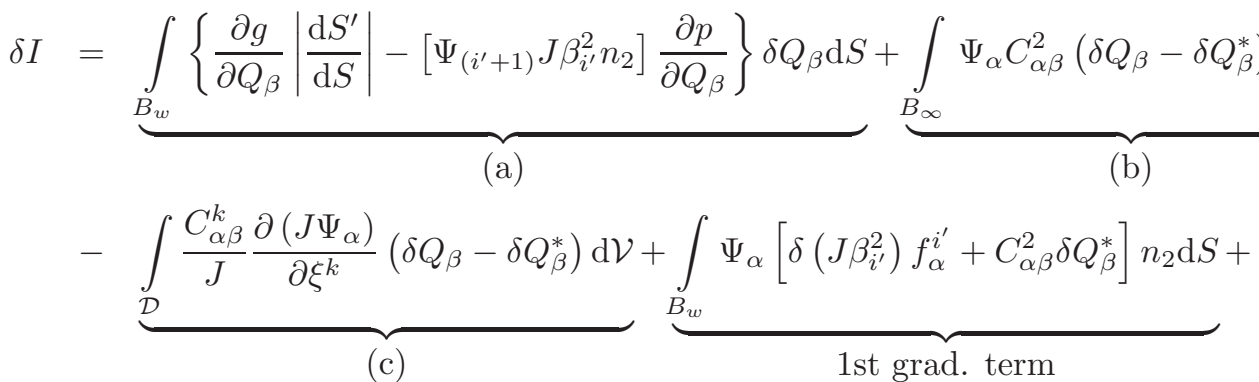

$$
\begin{aligned}
& +\underbrace{\int_{B_{w}}\left\{g \delta\left|\frac{\mathrm{d} S^{\prime}}{\mathrm{d} S}\right|+p\left[\Psi_{\left(i^{\prime}+1\right)} \delta\left(J \beta_{i^{\prime}}^{2}\right) n_{2}\right]\right\} \mathrm{d} S} \\
& \text { 2nd grad. term }
\end{aligned}
$$

Quite different from (19), in eq. (20) the sensitivity gradient amounts to just two integrals over the body surface $B_{w}$. The gradient integrals over $\mathcal{D}$ and $B_{\infty}$ from the former equation have been removed, but an additional variation term, $\delta Q_{\beta}^{*}$, has appeared in their stead. It refers to variations in the state variables that are owed to mesh movement $\delta \xi^{k}$ alone, at a fixed boundary configuration. ${ }^{50}$ Despite their particular cause, $\delta \xi^{k}$, they are of the same nature as the original $\delta Q_{\beta}$, in that they must also satisfy the Euler equations. For simplicity, the symbol $\delta \bar{Q}_{\beta}$ will be used to denote the difference: $\delta \bar{Q}_{\beta}=\delta Q_{\beta}-\delta Q_{\beta}^{*}$.

\section{The Adjoint Euler Equation}

In order to remove all physical variations from eq. (20), the first three terms in eq. (20) should vanish. The conditions under which that can be accomplished define the adjoint problem. Moreover, term $c$ can be regarded as an inner product between $\delta \bar{Q}_{\beta}$ and a differential operator, which is applied to $\Psi_{\alpha}$. Since all physical variations must be realizable, but they are otherwise arbitrary, it follows that the only means of eliminating the product is to require that the term involving $\Psi_{\alpha}$ be identically zero over $\mathcal{D}$, as a whole. The procedure gives rise to the adjoint equation, in steady form. Its similarity to the steady form of the Euler eqs. (10) enables one to formulate the adjoint problem in a way that is similar to the flow problem.

It is well-known that the Euler equations hyperbolic character is what ultimately determines their boundary problem. It also sets the conditions for their being well-posed. On having the adjoint equation share in the same character, one may extend the similarity to the adjoint boundary problem, as well. That can be accomplished by simply postulating that $\Psi_{\alpha}$ depends on time in a way that is similar to $Q_{\beta}$. This step 
leads to the following adjoint PDEs:

$$
\begin{aligned}
\frac{\partial \Psi_{\alpha}}{\partial t}-\frac{C_{\beta \alpha}^{k}}{J} \frac{\partial\left(J \Psi_{\beta}\right)}{\partial \xi^{k}} & =0 \\
\frac{\partial Q_{\alpha}}{\partial t}+\frac{C_{\alpha \beta}^{k}}{J} \frac{\partial Q_{\beta}}{\partial \xi^{k}} & =0
\end{aligned}
$$

which are paired with the flow governing eqs. (22) for comparison. It is worth noting here that the flux Jacobian matrix is transposed in the adjoint equations (21). One may also add that, despite their appearance, the adjoint equations are not in divergence form. For simplicity, then, one can redefine the adjoint variable so as to take in the transformation Jacobian, $\psi_{\alpha} \equiv J \Psi_{\alpha}$. It lends the adjoint equations the following form:

$$
\begin{aligned}
\frac{\partial \psi_{\alpha}}{\partial t}-C_{\beta \alpha}^{k} \frac{\partial \psi_{\beta}}{\partial \xi^{k}} & =0 \\
\frac{\partial \boldsymbol{\psi}}{\partial t}-\left(\mathbf{C}^{T} \cdot \nabla\right) \boldsymbol{\psi} & =0
\end{aligned}
$$

where the symbolic notation has been introduced for future convenience.

Clearly, one is only interested in the steady state solution to eqs. (23). For that solution alone drives term $c$ in eq. (20) to zero. The point in casting the adjoint PDEs in this form is that, not only do they share in the hyperbolic character of the Euler equations, but they also have the same characteristics. Their major difference lies in the sign reversal of the adjoint characteristic velocities. Quite distinct from the Euler eqs., though, the adjoint PDEs are linear. Because the flux Jacobian matrices do not depend on $\psi_{\alpha}$.

\section{Adjoint Boundary Conditions}

Before proceeding with the derivation of boundary conditions, though, it is important to note that the adjoint eqs. (23) are generalized in either form. Therefore, they must hold in any coordinate system and, in particular, in the Cartesian. The same applies to the periodic condition that is imposed on cut-planes (16). On the other hand, the wall and far-field boundary conditions, represented, respectively, by terms (a) and (b) in eq. (20), have been written for a specific system, where both boundaries are mapped onto constant coordinate planes: $B_{w} \Rightarrow \xi^{2}=0$ and $B_{\infty} \Rightarrow \xi^{2}=1$, respectively. However, they can also be put in Cartesian coordinates, as the following equations show:

$$
\begin{gathered}
\Psi_{\left(i^{\prime}+1\right)} J \beta_{i^{\prime}}^{2} n_{2}=\left.\psi_{\left(i^{\prime}+1\right)} n_{i^{\prime}}\right|_{B_{w}}=\frac{\partial g}{\partial p}\left|\frac{\mathrm{d} S^{\prime}}{\mathrm{d} S}\right| \\
\int_{B_{\infty}} \Psi_{\alpha} C_{\alpha \beta}^{2} \delta \bar{Q}_{\beta} n_{2} \mathrm{~d} S=\int_{B_{\infty}} \psi_{\alpha} \beta_{i^{\prime}}^{2} A_{\alpha \beta}^{i^{\prime}} \delta \bar{Q}_{\beta} n_{2} \mathrm{~d} S=\left.0 \Rightarrow \psi_{\alpha}\left(A_{\alpha \beta}^{i^{\prime}} n_{i^{\prime}}\right) \delta \bar{Q}_{\beta}\right|_{B_{\infty}}=0
\end{gathered}
$$

That opens up the possibility of solving the adjoint problem in the Cartesian system. It all hinges upon one's ability to compute the metric variations $\delta\left(J \beta_{i^{\prime}}^{2}\right)$ that appear in the sensitivity gradient (20), without having to resort to the transformation explicitly. If that is accomplished, then the transformation has fulfilled its prime objective, which was to simplify the derivation, and can be discarded.

For 2-D cases, the Greek subscripts range from 1 to 4 , where 1 refers to continuity, 2 and 3, to linear momentum and 4, to energy. Whereas the Latin indices refer to the two dimensions in physical space - for simplicity, we designate them as: $x^{1^{\prime}}=x, x^{2^{\prime}}=y$. The corresponding flux Jacobian matrices are denoted by: $\mathbf{A}^{1^{\prime}}=\mathbf{A}$ and $\mathbf{A}^{2^{\prime}}=\mathbf{B}$, respectively. Under these circumstances, the adjoint equations become

$$
\frac{\partial \psi_{\alpha}}{\partial t}-A_{\beta \alpha} \frac{\psi_{\beta}}{\partial x}-B_{\beta \alpha} \frac{\psi_{\beta}}{\partial y}=0
$$

For simplicity, the flux Jacobian vector ${ }^{52} A_{\beta \alpha}^{i^{\prime}}$ is also assigned symbolic notation, $A_{\beta \alpha}^{i^{\prime}} \Rightarrow \overline{\mathbf{A}}=\left(\mathbf{A}^{T}, \mathbf{B}^{T}\right)$.

It is widely known that the flux Jacobian matrices have complete eigensystems, and that they can be diagonalized, upon their being associated with a given direction. ${ }^{52,53}$ The normal direction to a boundary is of particular interest, in that regard. For the characteristics associated with it are responsible for all 
the exchange of information across that boundary. For a general treatment of such boundaries, we pick an arbitrary direction, as defined by the unit vector $\mathbf{k}=\left(k_{x}, k_{y}\right)^{T}$, and the corresponding Jacobian matrix is given by the dot product,

$$
-\overline{\mathbf{A}} \cdot \mathbf{k}=-k_{x} \mathbf{A}^{T}-k_{y} \mathbf{B}^{T}
$$

where the minus sign and the transposition, which appear in eqs. (26), have been retained for future convenience. The similarity transformation that diagonalizes the resulting matrix yields

$$
-\boldsymbol{\Lambda}=-\mathbf{P}^{T}(\overline{\mathbf{A}} \cdot \mathbf{k})\left(\mathbf{P}^{-1}\right)^{T}=\left(\begin{array}{cccc}
-\left(k_{x} u+k_{y} v\right) & 0 & 0 & 0 \\
0 & -\left(k_{x} u+k_{y} v\right) & 0 & 0 \\
0 & 0 & -\left(k_{x} u+k_{y} v+c\right) & 0 \\
0 & 0 & 0 & -\left(k_{x} u+k_{y} v-c\right)
\end{array}\right)
$$

which are obviously the same eigenvalues of the Euler equations, but for the sign reversal. Also associated with the direction $\mathbf{k}$, the similarity transformation operators are given by: ${ }^{52}$

$$
\begin{aligned}
\mathbf{P}^{T}= & \left(\begin{array}{cccc}
1 & u & v & \frac{u^{2}+v^{2}}{2} \\
0 & \rho k_{y} & -\rho k_{x} & \rho\left(u k_{y}-v k_{x}\right) \\
\frac{\rho}{2 c} & \frac{\rho\left(c k_{x}+u\right)}{2 c} & \frac{\rho\left(c k_{y}+v\right)}{2 c} & \frac{\rho}{2}\left(\frac{c}{\gamma-1}+u k_{x}+v k_{y}+\frac{u^{2}+v^{2}}{2 c}\right) \\
\frac{\rho}{2 c} & \frac{\rho\left(v-c k_{x}\right)}{2 c} & \frac{\rho\left(v-c k_{y}\right)}{2 c} & \frac{\rho}{2}\left(\frac{c}{\gamma-1}-u k_{x}-v k_{y}+\frac{u^{2}+v^{2}}{2 c}\right)
\end{array}\right) \\
\left(\mathbf{P}^{-1}\right)^{T}= & \left(\begin{array}{ccccc}
1-\frac{(\gamma-1)\left(u^{2}+v^{2}\right)}{2 c^{2}} & \frac{v k_{x}-u k_{y}}{\rho} & \frac{(\gamma-1)\left(u^{2}+v^{2}\right)-2 c\left(u k_{x}+v k_{y}\right)}{2 \rho c} & \frac{(\gamma-1)\left(u^{2}+v^{2}\right)+2 c\left(u k_{x}+v k_{y}\right)}{2 \rho c} \\
\frac{\frac{(\gamma-1) u}{c^{2}}}{\frac{(\gamma-1) v}{c^{2}}} & \frac{1}{\rho} k_{y} & \frac{c k_{x}-(\gamma-1) u}{\rho c} & \frac{-c k_{x}-(\gamma-1) u}{\rho c} \\
-\frac{(\gamma-1)}{c^{2}} & 0 & \frac{c k_{y}-(\gamma-1) v}{\rho c} & \frac{-c k_{y}-(\gamma-1) v}{\rho c} \\
\rho c & \frac{\gamma-1}{\rho c}
\end{array}\right)
\end{aligned}
$$

respectively.

Although the adjoint PDEs cannot be fully diagonalized, one can obtain their characteristic form by left multiplying eq. (26) through by $\mathbf{P}^{T}$. It leads to the expression

$$
\mathbf{P}^{T} \frac{\partial \boldsymbol{\psi}}{\partial t}-\mathbf{P}^{T} \mathbf{A}\left(\mathbf{P}^{-1}\right)^{T} \mathbf{P}^{T} \frac{\partial \boldsymbol{\psi}}{\partial x}-\mathbf{P}^{T} \mathbf{B}\left(\mathbf{P}^{-1}\right)^{T} \mathbf{P}^{T} \frac{\partial \boldsymbol{\psi}}{\partial y}=0
$$

Accordingly, on computing the matrix products in eq. (31) one obtains a set of Riemann's equations, or characteristics, of the form:

$$
\sum_{\beta=1}^{N} K_{\alpha \beta}^{t} \frac{\partial \psi_{\beta}}{\partial t}=\sum_{\beta=1}^{N} K_{\alpha \beta}^{x} \frac{\partial \psi_{\beta}}{\partial x}+\sum_{\beta=1}^{N} K_{\alpha \beta}^{y} \frac{\partial \psi_{\beta}}{\partial y}
$$

where $N=4$ in the $2-\mathrm{D}$ cases and the coefficients $K_{\alpha \beta}^{t}, K_{\alpha \beta}^{x}$ and $K_{\alpha \beta}^{y}$ are listed in table 1 , at the end of the paper.

Just as the original eqs. (26), the set (32) is linear. Only, the coefficients are given by less familiar, more complex, algebraic expressions. Different from the former, though, each one of the above equations $(\alpha)$ is intrinsically associated with a specific characteristic velocity, as given by the matrix $\Lambda$ in eq. (28). Ultimately, on choosing the vector $\mathbf{k}$ normal to the domain boundaries, those velocities determine which of the adjoint characteristics enter or leave the flow domain.

In a sense, the state variables can be seen as degrees of freedom (DOFs) of the boundary problem. Naturally, their number corresponds to the dimension $N$ of the state space. In $2-\mathrm{D}$ problems, $N=4$. In this context, each boundary condition constrains one DOF at the boundary. Whereas each outgoing characteristic corresponds to a DOF that is preserved. The scheme holds for both problems, the Euler flow and its adjoint. However, in view of the sign reversal, to each DOF that is constrained for the flow at a boundary, there corresponds one that is left for the adjoint, and vice-versa. 


\section{IV.A. Solid Wall}

The 2-D contour of the body can be specified as a level curve, such as $\eta(x, y)=0$, in a plane mapping of type: $(x, y) \rightleftharpoons(\xi, \eta)$. Alternatively, it can be cast in parametric form as

$$
\eta(x, y)=0 \Leftrightarrow\left\{\begin{array}{l}
x=x(\xi, 0) \\
y=y(\xi, 0)
\end{array}\right.
$$

In the first case, the normal unit vector, can be obtained by simply making $\mathbf{n}=\nabla \eta /\|\nabla \eta\|$. The corresponding expression for the second case may be derived from the first by means of the relations ${ }^{\mathrm{a}}$ $\eta_{, x}=-y_{, \xi} / J$ and $\eta_{, y}=x_{, \xi} / J$, which are gotten on inverting the operator $\beta_{j^{\prime}}^{i}$ to compute $\beta_{k}^{i^{\prime}}$. As a result, the normal vector becomes

$$
n_{j^{\prime}} \Rightarrow\left(\begin{array}{c}
k_{x} \\
k_{y}
\end{array}\right)=\frac{1}{\sqrt{\eta_{, x}^{2}+\eta_{, y}^{2}}}\left(\begin{array}{c}
\eta_{, x} \\
\eta_{, y}
\end{array}\right)=\frac{1}{\sqrt{x_{, \xi}^{2}+y_{, \xi}^{2}}}\left(\begin{array}{c}
-y_{, \xi} \\
x_{, \xi}
\end{array}\right)
$$

in the Cartesian system. The term that multiplies the vector on the RHS can be recognized to be an element of the metric tensor $g_{i j}$ in transformed space: $g_{11}=x_{, \xi}^{2}+y_{, \xi}^{2}$. In any case, the result yields a fairly simple expression for the vector covariant components in the transformed plane.

$$
n_{i}=n_{j^{\prime}} \beta_{i}^{j^{\prime}}=\frac{1}{\sqrt{g_{11}}}\left(\begin{array}{c}
0 \\
J
\end{array}\right)
$$

Before proceeding with the derivation, it should be noted that both $\mathbf{n}$ and the metric term $g_{11}$ can be evaluated at the wall, solely on the basis of the parametric representation of the contour.

The wall boundary condition is given by eq. (24). It depends on the specification of a metric term, namely the area element ratio $\left|\mathrm{d} S^{\prime} / \mathrm{d} S\right|$. In parametric form (33), one has $\mathrm{d} S=\mathrm{d} \xi$. Besides, $\mathrm{d} S^{\prime}$ and $\mathrm{d} S$ are clearly parallel: $\mathrm{d} S^{\prime} \| \mathrm{d} S$. However, the ratio between the two of them comes from the arc length element ${ }^{54,55}$

$$
\left|\frac{\mathrm{d} S^{\prime}}{\mathrm{d} S}\right| \mathrm{d} S=\left|\frac{\mathrm{d} S^{\prime}}{\mathrm{d} \xi}\right| \mathrm{d} \xi=\sqrt{1+\left(\frac{\mathrm{d} y}{\mathrm{~d} x}\right)_{B_{w}}^{2}} x_{, \xi} \mathrm{d} \xi=\sqrt{x_{, \xi}^{2}+y_{, \xi}^{2}} \mathrm{~d} \xi=\sqrt{g_{11}} \mathrm{~d} \xi
$$

Hence the solid wall boundary condition simply becomes:

$$
k_{x} \psi_{2}+k_{y} \psi_{3}=\frac{\partial g}{\partial p} \sqrt{g_{11}}
$$

As was discussed above, the adjoint solution is computed on the basis of a stationary flow-field. Therefore, by differentiating eq. (37) with respect to time, one gets,

$$
k_{x} \frac{\partial \psi_{2}}{\partial t}+k_{y} \frac{\partial \psi_{3}}{\partial t}=0
$$

This expression is used next, to simplify the equation of the characteristic that leaves the domain through the solid wall boundary.

The normal velocity vanishes identically at the wall $\left(k_{x} u+k_{y} v=0\right)$, which implies that the first two characteristic velocities in eq. (28) are zero. They cannot transfer any Cauchy data across that boundary. On the other hand, on choosing the normal vector pointing inward, to the domain, the third and fourth characteristics become $-c$ and $+c$, respectively. Therefore, the third one leaves the domain, while the fourth one enters it. In view of these results, the third characteristic equation in (32) should be solved for the adjoint variables at the wall, whereas the fourth one should be replaced by condition (37). Two DOFs still remain, because of the first and second characteristics. In principle, then, these should be extrapolated from the domain.

On taking the third characteristic from (32) and factoring the zero normal velocity in it, as well as eq. (38), one obtains a simplified form of the equation that should be solved for the $\psi_{k}$ at the wall. The equation

${ }^{\mathrm{a}} \mathrm{A}$ shorthand notation is used here to denote partial derivatives of metric terms: ()$_{, \xi}=\partial() / \partial \xi$ and ()$_{, x}=\partial() / \partial x$. 
reads:

$$
\begin{aligned}
\frac{\partial \psi_{1}}{\partial t}+u \frac{\partial \psi_{2}}{\partial t}+v \frac{\partial \psi_{3}}{\partial t}+h_{o} \frac{\partial \psi_{4}}{\partial t} & =\left(u+k_{x} c\right) \frac{\partial \psi_{1}}{\partial x}+\left(v+k_{y} c\right) \frac{\partial \psi_{1}}{\partial y}+\left[c^{2} k_{y}^{2}+\left(c k_{x}+u\right)^{2}\right] \frac{\partial \psi_{2}}{\partial x}+ \\
& -\left[c^{2} k_{x} k_{y}-\left(c k_{x}+u\right)\left(c k_{y}+v\right)\right]\left(\frac{\partial \psi_{2}}{\partial y}+\frac{\partial \psi_{3}}{\partial x}\right)+ \\
& +\left[c^{2} k_{x}^{2}+\left(c k_{y}+v\right)^{2}\right] \frac{\partial \psi_{3}}{\partial y}-\left[c^{2} k_{y}\left(k_{x} v-k_{y} u\right)-\left(u+k_{x} c\right) h_{o}\right] \frac{\partial \psi_{4}}{\partial x}+ \\
& -\left[c^{2} k_{x}\left(k_{y} u-k_{x} v\right)-\left(v+k_{y} c\right) h_{o}\right] \frac{\partial \psi_{4}}{\partial y}
\end{aligned}
$$

where the symbol $h_{o}$ stands for the specific stagnation enthalpy.

Equations (39) and (37) represent all the Cauchy data that crosses the solid wall. Together, they should form the set of Riemann equations at that boundary. The problem with that set is the presence of all four adjoint variables in eq. (39). Had the simplified characteristic equation been rid of $\psi_{1}$ and $\psi_{4}$, then one would have a closed set to solve for $\psi_{2}$ and $\psi_{3}$. In that case, $\psi_{1}$ and $\psi_{4}$ would not be part of the Cauchy problem at the wall and, thus, they would be determined by numerical extrapolation, alone. The situation would, then, be similar to the corresponding flow boundary problem.

As it stands, though, the set cannot be solved for the $\psi_{k}$ at the wall. Instead, one must rely on eq. (37) and an extrapolation scheme. In that regard, the aforementioned Reuther's ${ }^{49}$ scheme suits our applications very well. It adopts zero order extrapolations for $\psi_{1}$ and $\psi_{4}$ and divides (37) into two conditions between $\psi_{2}$ and $\psi_{3}$, so that their sum recovers the original equation. On representing ghost cells values by $\psi_{k}^{-}$and the corresponding values in domain neighboring cells by $\psi_{k}^{+}$, the equations read

$$
\left\{\begin{array}{l}
\psi_{1}^{-}=\psi_{1}^{+} \\
\psi_{2}^{-}=\psi_{2}^{+}+\frac{2 k_{x}}{k_{x}^{2}+k_{y}^{2}}\left(\frac{\partial g}{\partial p} \sqrt{g_{11}}-k_{x} \Psi_{2}^{+}-k_{y} \Psi_{3}^{+}\right) \\
\psi_{3}^{-}=\psi_{3}^{+}+\frac{2 k_{y}}{k_{x}^{2}+k_{y}^{2}}\left(\frac{\partial g}{\partial p} \sqrt{g_{11}}-k_{x} \Psi_{2}^{+}-k_{y} \Psi_{3}^{+}\right) \\
\psi_{4}^{-}=\psi_{4}^{+}
\end{array}\right.
$$

where the condition (37) is met at the wall boundary in terms of the average,

$$
k_{x} \frac{\left(\psi_{2}^{-}+\psi_{2}^{+}\right)}{2}+k_{y} \frac{\left(\psi_{3}^{-}+\psi_{3}^{+}\right)}{2}=\frac{\partial g}{\partial p} \sqrt{g_{11}}
$$

This scheme has been amply verified in the literature, and by ourselves. ${ }^{51,56}$ It has shown to lead to estimates of the sensitivity gradient that are close to those obtained by finite differences, within reasonable accuracy levels. ${ }^{49}$ Therefore, it is fully consistent with what is expected of the adjoint method.

\section{IV.B. Entrance}

On choosing the normal vector pointing inward, to the domain, at a subsonic inflow boundary, there is only one adjoint characteristic that enters the flow domain. It is the one that is associated with the eigenvalue $-(\mathbf{u} \cdot \mathbf{n}-c)$ in eq. (28), that is the fourth PDE in (32). Hence that equation must be replaced by a boundary condition, which should come from eq. (25), as a requirement of realizability.

Together with the first three PDEs from set (32), this condition forms the Riemann problem at that boundary. In order to derive it, one must first find out which virtual displacements $\delta \bar{Q}_{\beta}$ are admissible there.

At a subsonic entrance, the flow boundary conditions require that the velocity direction $\theta$, along with the stagnation pressure $p_{o}$ and temperature $T_{o}$, be prescribed. It clearly implies that the corresponding variations are null: $\delta \theta=\delta p_{o}=\delta T_{o}=0$. In terms of state variables $Q_{\beta}$, these quantities are given by:

$$
\left\{\begin{array}{l}
\tan (\theta)=\frac{Q_{3}}{Q_{2}} \\
T_{o}=\frac{(\gamma-1)}{2 \gamma R Q_{1}^{2}}\left[2 \gamma Q_{1} Q_{4}-(\gamma-1)\left(Q_{2}^{2}+Q_{3}^{2}\right)\right] \\
p_{o}=\frac{(\gamma-1)\left[2 Q_{1} Q_{4}-\left(Q_{2}^{2}+Q_{3}^{2}\right)\right]}{2 Q_{1}}\left\{1+\frac{\left(Q_{2}^{2}+Q_{3}^{2}\right)}{\gamma\left[2 Q_{1} Q_{4}-\left(Q_{2}^{2}+Q_{3}^{2}\right)\right]}\right\}^{\frac{\gamma}{(\gamma-1)}}
\end{array}\right.
$$


By computing $\delta \theta, \delta p_{o}$ and $\delta T_{o}$ in terms of the variations $\delta Q_{\beta}$, and by equating them to zero, one gets a linear set of three equations. Here, it must be recalled that $\delta Q_{\beta}$ and $\delta \bar{Q}_{\beta}$ are equivalent with regard to realizability. Then on replacing the former by the latter, one can solve the set for three of them in terms of the fourth one. Whichever variation is chosen as the independent one, the resulting expressions will represent the locus of realizability in state space. For instance, on picking $\delta \bar{Q}_{1}$ to be independent, the procedure yields:

$$
\delta \bar{Q}_{\beta} \Rightarrow\left(\begin{array}{c}
1 \\
\frac{u}{2}\left[2-\gamma+\gamma^{2}-\frac{2 e_{t}(\gamma-1) \gamma}{\left(u^{2}+v^{2}\right)}\right] \\
\frac{v}{2}\left[2-\gamma+\gamma^{2}-\frac{2 e_{t}(\gamma-1) \gamma}{\left(u^{2}+v^{2}\right)}\right] \\
\frac{1}{2}\left[-2 e_{t}(\gamma-2) \gamma+(\gamma-1)^{2}\left(u^{2}+v^{2}\right)\right]
\end{array}\right) \delta \bar{Q}_{1}
$$

Then, on substituting vector (43) for the corresponding term in eq. (25) one obtains the equation

$$
\delta Q_{1}\left\{C_{1} \psi_{1}+C_{2} \psi_{2}+C_{3} \psi_{3}+C_{4} \psi_{4}\right\}=0
$$

where the coefficients are given by:

$$
\left\{\begin{array}{l}
C_{1}=\left[2-\gamma+\gamma^{2}-\frac{2 \gamma e_{t}(\gamma-1)}{u^{2}+v^{2}}\right] \frac{\left(k_{x} u+k_{y} v\right)}{2} \\
C_{2}=\frac{\left(2-\gamma+\gamma^{2}\right) k_{x} u^{2}+2\left(1-\gamma+\gamma^{2}\right) k_{y} u v}{2}-\frac{(\gamma-1) \gamma k_{x} v^{2}}{2}-\frac{2 \gamma e_{t}(\gamma-1)\left[2 k_{y} u v+k_{x}\left(u^{2}-v^{2}\right)\right]}{2\left(u^{2}+v^{2}\right)} \\
C_{3}=\frac{-\gamma(\gamma-1) k_{y} u^{4}+2 k_{y} u^{2} v^{2}}{2\left(u^{2}+v^{2}\right)}+\frac{2\left(1-\gamma+\gamma^{2}\right) k_{x}\left(u^{3} v+u v^{3}\right)+\left(2-\gamma+\gamma^{2}\right) k_{y} v^{4}+2 \gamma e_{t}(\gamma-1)\left[k_{y}\left(u^{2}-v^{2}\right)-2 k_{x} u v\right]}{2\left(u^{2}+v^{2}\right)} \\
C_{4}=-\frac{\left(k_{x} u+k_{y} v\right)\left[(\gamma-1)\left(u^{2}+v^{2}\right)-2 \gamma e_{t}\right]\left[\left(2-\gamma+\gamma^{2}\right)\left(u^{2}+v^{2}\right)-2 \gamma e_{t}(\gamma-1)\right]}{4\left(u^{2}+v^{2}\right)}
\end{array}\right.
$$

In eq. (44), $\delta \bar{Q}_{1}$ is realizable, but it is otherwise arbitrary. Therefore, the only means for one to drive the equation to zero is to require its coefficient, i.e. the term within braces, to vanish identically. That leads to a single linear equation, which involves all four $\psi_{\alpha}$ :

$$
C_{1} \psi_{1}+C_{2} \psi_{2}+C_{3} \psi_{3}+C_{4} \psi_{4}=0
$$

Eq. (46) is the boundary condition to be imposed on the adjoint variables, and it may also be seen as a compatibility relation they should meet at the inflow boundary. Since the adjoint problem is based on a stationary flow solution, the coefficients in (45) are independent of time. Therefore, eq. (46) remains valid when the $\psi_{\alpha}$ are differentiated with respect to time. Then, on joining it with the first three characteristic equations from (32), one obtains the complete linear set for the corresponding Riemann problem.

$$
\left\{\begin{array}{l}
K_{11}^{t} \frac{\partial \psi_{1}}{\partial t}+K_{12}^{t} \frac{\partial \psi_{2}}{\partial t}+K_{13}^{t} \frac{\partial \psi_{3}}{\partial t}+K_{14}^{t} \frac{\partial \psi_{4}}{\partial t}=R_{1} \\
K_{21}^{t} \frac{\partial \psi_{1}}{\partial t}+K_{22}^{t} \frac{\partial \psi_{2}}{\partial t}+K_{23}^{t} \frac{\partial \psi_{3}}{\partial t}+K_{24}^{t} \frac{\partial \psi_{4}}{\partial t}=R_{2} \\
K_{31}^{t} \frac{\partial \psi_{1}}{\partial t}+K_{32}^{t} \frac{\partial \psi_{2}}{\partial t}+K_{33}^{t} \frac{\partial \psi_{3}}{\partial t}+K_{34}^{t} \frac{\partial \psi_{4}}{\partial t}=R_{3} \\
C_{1} \frac{\partial \psi_{1}}{\partial t}+C_{2} \frac{\partial \psi_{2}}{\partial t}+C_{3} \frac{\partial \psi_{3}}{\partial t}+C_{4} \frac{\partial \psi_{4}}{\partial t}=0
\end{array}\right.
$$

where the spatial derivatives from the RHS of (32) have all been collected up in the $R_{\alpha}$ terms. The resulting set is amenable to numerical integration by explicit time stepping.

With regard to the discussion on boundary conditions, it is worth noting that, for each one of the three DOFs that are constrained in the flow problem, a corresponding DOF is preserved in the adjoint problem. That symmetry agrees exactly with the balance between the domain incoming and outgoing characteristics for both problems. 


\section{IV.C. Exit}

In the case of an outflow boundary, there are three domain incoming characteristics. On choosing the normal vector pointing inward, to the domain, they are the ones that correspond to the first two and the fourth eigenvalues in (28), and their equations in (32) must be replaced by boundary conditions. Hence, it is only the characteristic associated with $-(\mathbf{u} \cdot \mathbf{n}+c)$ that needs actually be solved.

Just as in the previous case, the boundary conditions should come as a result of realizability constraints that are imposed on $\delta \bar{Q}_{\alpha}$. In the applications of interest here, the static pressure is the only quantity that is fixed at an outflow boundary. On casting it in terms of the state variables, it yields

$$
p=(\gamma-1)\left(Q_{4}-\frac{\left(Q_{2}^{2}+Q_{3}^{2}\right)}{2 Q_{1}}\right)
$$

By computing its first variation and setting it at zero $(\delta p=0)$, and on recalling that, as far as realizability is concerned, $\delta \bar{Q}_{\alpha} \Leftrightarrow \delta Q_{\alpha}$, one gets

$$
\delta \bar{Q}_{\alpha} \Rightarrow\left(\begin{array}{c}
\delta \bar{Q}_{1} \\
\delta \bar{Q}_{2} \\
\delta \bar{Q}_{3} \\
u \delta \bar{Q}_{2}+v \delta \bar{Q}_{3}-\frac{\left(u^{2}+v^{2}\right)}{2} \delta \bar{Q}_{1}
\end{array}\right)
$$

The above vector (49) represents the locus of realizable variations for the outflow boundary. Again, on substituting it for the corresponding variations in eq. (25), it leads to:

$$
\begin{aligned}
& \delta Q_{1}\left\{\left(k_{x} u+k_{y} v\right)\left(-u \psi_{2}-v \psi_{3}\right)+\frac{\left(k_{x} u+k_{y} v\right)}{2} \psi_{4}\left[(\gamma-2)\left(u^{2}+v^{2}\right)-2 \gamma e_{t}\right]\right\}+ \\
+ & \delta Q_{2}\left\{k_{x}\left(\psi_{1}+v \psi_{3}\right)+\psi_{2}\left(u+k_{x} u+k_{y} v\right)+\psi_{4}\left[k_{x} \gamma e_{t}+u\left(k_{x} u+k_{y} v\right)+\frac{(\gamma-1) k_{x}\left(u^{2}+v^{2}\right)}{2}\right]\right\}+ \\
+ & \delta Q_{3}\left\{k_{y}\left(\psi_{1}+u \psi_{2}\right)+\psi_{3}\left(v+k_{x} u+k_{y} v\right)+\psi_{4}\left[k_{y} \gamma e_{t}+v\left(k_{x} u+k_{y} v\right)-\frac{(\gamma-1) k_{y}\left(u^{2}+v^{2}\right)}{2}\right]\right\}=0
\end{aligned}
$$

Naturally, the $\delta \bar{Q}_{1}, \delta \bar{Q}_{2}$ and $\delta \bar{Q}_{3}$ are arbitrary, albeit realizable. Therefore, the only means of driving eq. (50) to zero is to require their coefficients to vanish identically. That leads to three boundary conditions, involving all four $\psi_{\alpha}$. They can be written in a simplified form as:

$$
\left\{\begin{array}{l}
\psi_{1}=\frac{1}{2}\left[2 e_{t} \gamma-(\gamma-1)\left(u^{2}+v^{2}\right)\right] \psi_{4}=C_{1} \psi_{4} \\
\psi_{2}=\left[\frac{-2 e_{t} \gamma k_{x}+(\gamma-2) k_{x} u^{2}-2 k_{y} u v+\gamma k_{x} v^{2}}{2\left(k_{x} u+k_{y} v\right)}\right] \psi_{4}=C_{2} \psi_{4} \\
\psi_{3}=\left[\frac{-2 e_{t} \gamma k_{y}+(\gamma-2) k_{y} v^{2}-2 k_{x} u v+\gamma k_{y} u^{2}}{2\left(k_{x} u+k_{y} v\right)}\right] \psi_{4}=C_{3} \psi_{4}
\end{array}\right.
$$

Just as in the previous case, all of the above coefficients are based on a steady flow solution and, thus, they are independent of time. As a result, these equations still hold when the $\psi_{\alpha}$ are differentiated with respect to time.

The complete set for the Riemann problem at the outflow boundary is, then, obtained by assembling the above (51) with the equation of the third characteristic from (32)

$$
\begin{cases}\frac{\partial \psi_{1}}{\partial t}-C_{1} \frac{\partial \psi_{4}}{\partial t} & =0 \\ \frac{\partial \psi_{2}}{\partial t}-C_{2} \frac{\partial \psi_{4}}{\partial t} & =0 \\ \frac{\partial \psi_{3}}{\partial t}-C_{3} \frac{\partial \psi_{4}}{\partial t} & =0 \\ \left(\sum_{\beta=1}^{3} K_{3 \beta}^{t} C_{\beta}+K_{34}^{t}\right) \frac{\partial \psi_{4}}{\partial t} & =R_{3}\end{cases}
$$


Similar to the previous case, the spatial derivatives in the RHS of the third eq. from (32) have been collected up in the $R_{3}$ term. The resulting set is also amenable to numerical integration by explicit time stepping.

With respect to the relation between the flow and adjoint boundary conditions, the situation here is similar to the previous case too. For a single DOF is constrained in the flow problem and single DOF is preserved in the adjoint. Here again, the symmetry agrees exactly with the balance between the domain incoming and outgoing characteristics for both problems.

\section{Results}

In order to validate the above treatment of adjoint boundary conditions, it would be instructive to pursue a two-steps procedure. First, the idea is to check whether it leads to correct results in a complete application. It may well be an inverse design application, where the target geometry is known beforehand, as it was done in a previous paper. ${ }^{47}$ Then one ought to compare adjoint solutions that this treatment yields to those that are obtained under homogeneous boundary conditions at the far-field. An usual definition of objective functional for inverse design applications is given by the mean square error of the actual pressure distribution $p$ with respect to a target distribution $p_{d}$,

$$
I=\frac{1}{2} \int_{B_{w}}\left(p-p_{d}\right)^{2} \mathrm{~d} s
$$

The target pressure distribution $p_{d}$ in the validation test has been obtained from the flow solution around a known geometry, on making use of the same flow solver and geometry representation. The procedure ensures that not only is the target realizable, but it is also attainable within the current framework.

Flow and adjoint solutions alike were computed on unstructured meshes with triangular elements. The far-field boundary is placed over 50 chord lengths away from the airfoil surface. All geometries have been represented by the CST (Class Shape-function Transformation) parameterization. ${ }^{57,58}$ Numerical simulations for the adjoint and flow equations have been run with a cell-centered Finite Volume Method, by using the $2^{\text {nd }}$ order 5 -step Runge-Kutta time-stepping scheme. ${ }^{59}$

For simplicity, the steepest descent method was adopted as the optimization procedure. The inverse design loop consists of the following sequence: mesh generation for a given geometry, flow simulation, adjoint solution, evaluation of sensitivity gradient, and gradient based changes to geometry. The cycles are repeated until a local extremum of (53) is reached, within a prescribed accuracy level.

A test case is presented next, where the initial geometry is a RAE 2822 at $M_{\infty}=0.75$ and an angle of attack of $\alpha=0^{\circ}$. The target $p_{d}$ corresponds to those of a NACA 0012 under the same flow conditions. Figure 1 shows the initial and final geometries with corresponding pressure distributions.
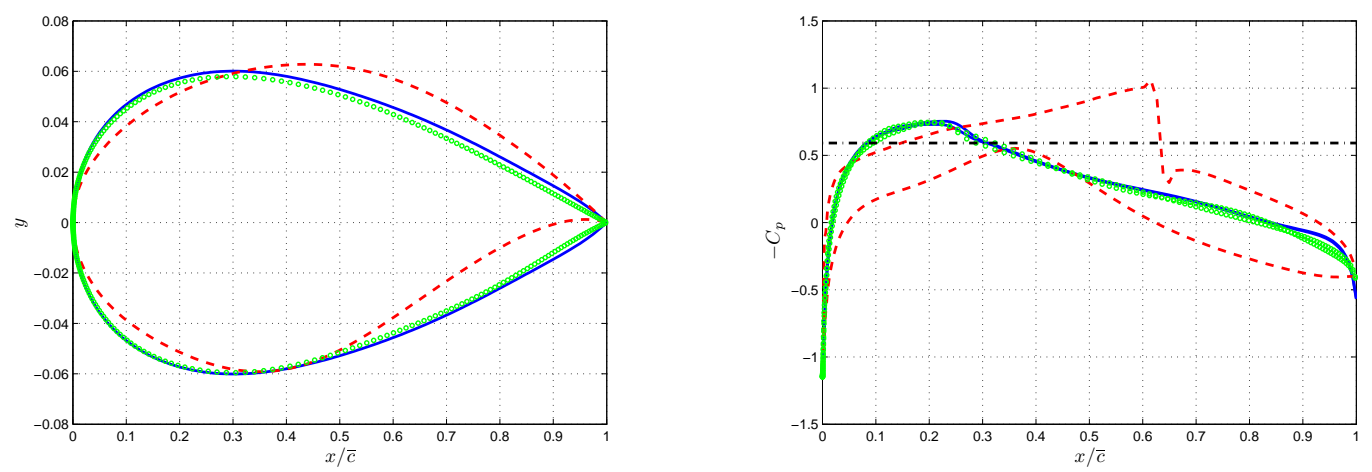

Figure 1. Mach 0.75 and $\alpha=0^{\circ}$. Left, geometries. Right, $C_{p}$. Red dash lines, original curves; green cicles, final results; blue solid lines, target profiles; black dash-dot, $C_{p}^{*}$.

As the above results show, the characteristics-based boundary conditions work properly. For the target geometry has been successfully recovered in the process: the differences between them remain below $3.5 \times$ $10^{-3}$. As was discussed above, the sensitivity gradient computation here makes use of the reduced expression. Therefore, it only requires the adjoint solution on the airfoil contour. Figures 2 and 3 present them, as they 
appear at the end of the first cycle, for the upper and lower sides of the airfoil, respectively. The left side of each figure shows characteristics-based results, whereas the right side depicts results from the homogeneous boundary conditions in each case.
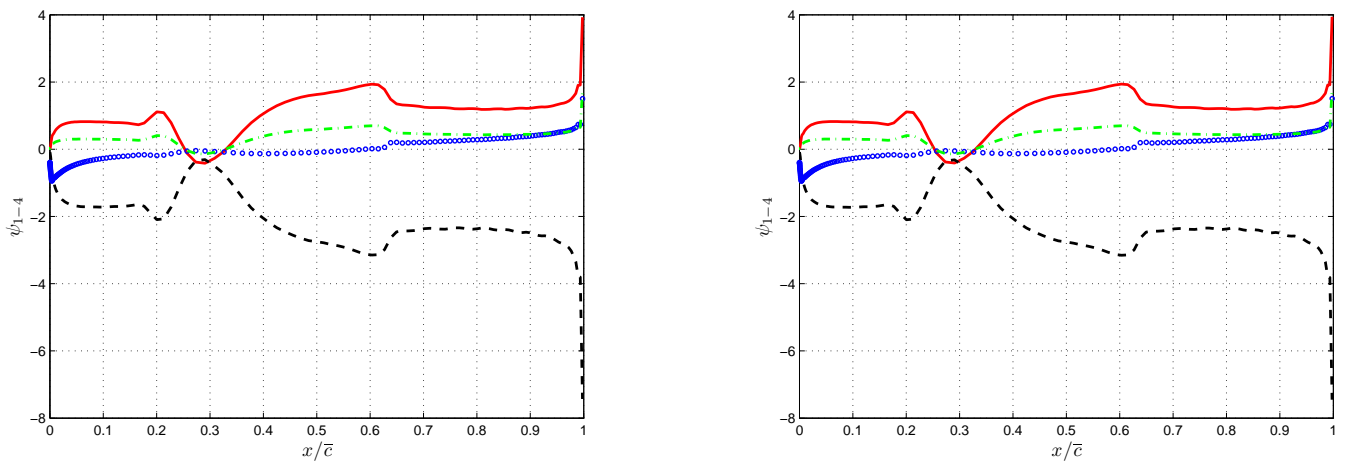

Figure 2. Airfoil upper side adjoint solution. Left, homogeneous boundary conditions. Right, characteristics-based boundary conditions. Red solid line, $\psi_{1}$; black dashed line, $\psi_{2}$; blue circles, $\psi_{3}$; green dash-dot, $\psi_{4}$.
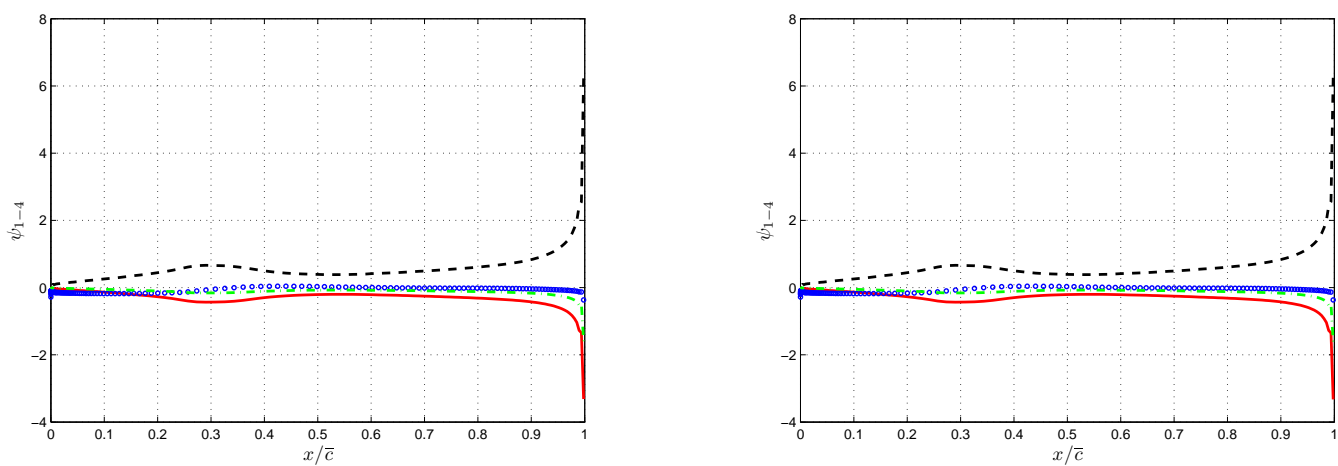

Figure 3. Airfoil lower side adjoint solution. Left, homogeneous boundary conditions. Right, characteristics-based boundary conditions. Red solid line, $\psi_{1}$; black dashed line, $\psi_{2}$; blue circles, $\psi_{3}$; green dash-dot, $\psi_{4}$.

The solutions have essentially the same character over the whole airfoil contour. They even share the same discontinuity at the trailing edge. However, there is a small, yet significant, difference between them. In all points other than in the immediate neighborhood of the singularity, the difference remains below $8 \times 10^{-3}$. That value is higher than the maximum residue of the adjoint solutions $\left(7 \times 10^{-5}\right)$, hence its significance. On the other hand, that value is apparently too small to cause any noticeable differences between the two approaches, in the estimates of the sensitivity gradient. In any case, the evidence suggests that further tests be devised to ascertain the influence the distance from the far-field boundary has on the adjoint solution at the contour.

Quite a different outcome is gotten for internal flows. Where one does not usually have the freedom to choose the position of permeable boundaries at will. In most cases, the proximity of those boundaries makes the full characteristics approach a necessity. In order to illustrate the problem, we have computed adjoint solutions for an internal flow case, by imposing homogenous and full characteristics conditions on the inflow and outflow boundaries. The same objective functional (53) was used, but the sole purpose was to solve the adjoint equations. After all, the reduced gradient form would not suit this case.

The set-up for the test is presented in fig. 4. The left side depicts initial and target geometries, while the respective $C_{p}$ distributions are shown on the right side - the latter are based on the nozzle length.

Figure 5 presents the adjoint solutions at the nozzle surface. The results for homogeneous boundary conditions are on the left side, whereas those on the right side represent the full characteristics approach.

As can be seen, the differences between the solutions are quite significant, even in terms of general character. Moreover, the homogenous conditions have led to discontinuities at the boundaries, which could only be negotiated by the solver at the cost of a substantial increase in artificial dissipation, locally. To further emphasize the point, fig. 6 depicts the solution for one of the adjoint variables, namely $\psi_{1}$, for both 

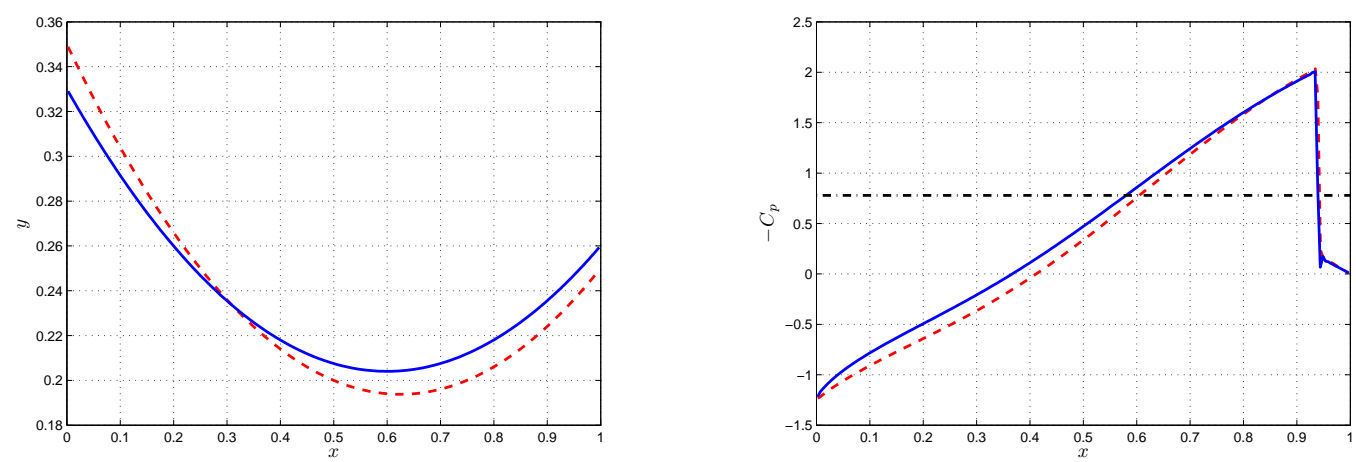

Figure 4. Mach 0.7. Left, geometries. Right, $C_{p}$. Red dashed line, original curves; blue solid line, target profiles; black dashdot, $C_{p}^{*}$.
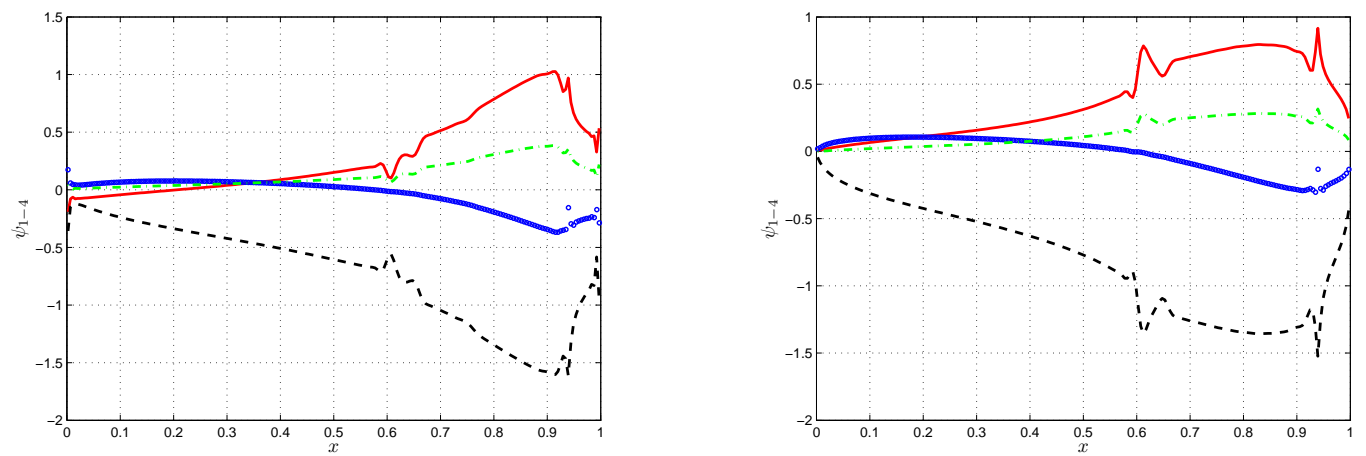

Figure 5. Left, homogeneous boundary conditions. Right, characteristics-based boundary conditions. Red solid line, $\psi_{1}$; black dashed line, $\psi_{2}$; blue circles, $\psi_{3}$; green dashdot, $\psi_{4}$.

boundary condition treatments. It clearly shows that the differences between them are not limited to the wall.

\section{Conclusions}

The main purpose of this work has been to investigate the adjoint boundary problem on the basis of its similarity to the corresponding flow problem. The rationale behind our approach was to make use of that similarity to seek conditions for the well-posedness of the adjoint equations. On accounting for the hyperbolic character the latter share with the Euler equations, an attempt was made to construct an adjoint Riemann problem at each boundary. Two aspects concur for that purpose:

First, the sign reversal of adjoint characteristics lends them a complementary quality with respect to those of the flow, in that there is a symmetry between the two problems, in the way Cauchy data is transferred across the boundaries.

Second, the boundary integrals that result from the use of Gauss' theorem are seen as inner products between adjoint variables and feasible variations of the flow physics. The conditions under which those products vanish determine the former as normal vectors to a locus of realizability in state space — we have termed them compatibility conditions.

The algebra has been shown to yield a balance between the compatibility conditions and the corresponding number of domain incoming characteristics. Therefore, one is able to put together a complete set of adjoint Riemann equations for each permeable boundary. The end result is a set of boundary conditions that is entirely similar to those that render the Euler equations well-posed. The solid wall is the only exception. For there are characteristics that do not cross the wall and, hence, they cannot transfer Cauchy data across it. The adjoint Riemann set is indeterminate in this case, and one must rely on the boundary extrapolation schemes that are found in the literature. 

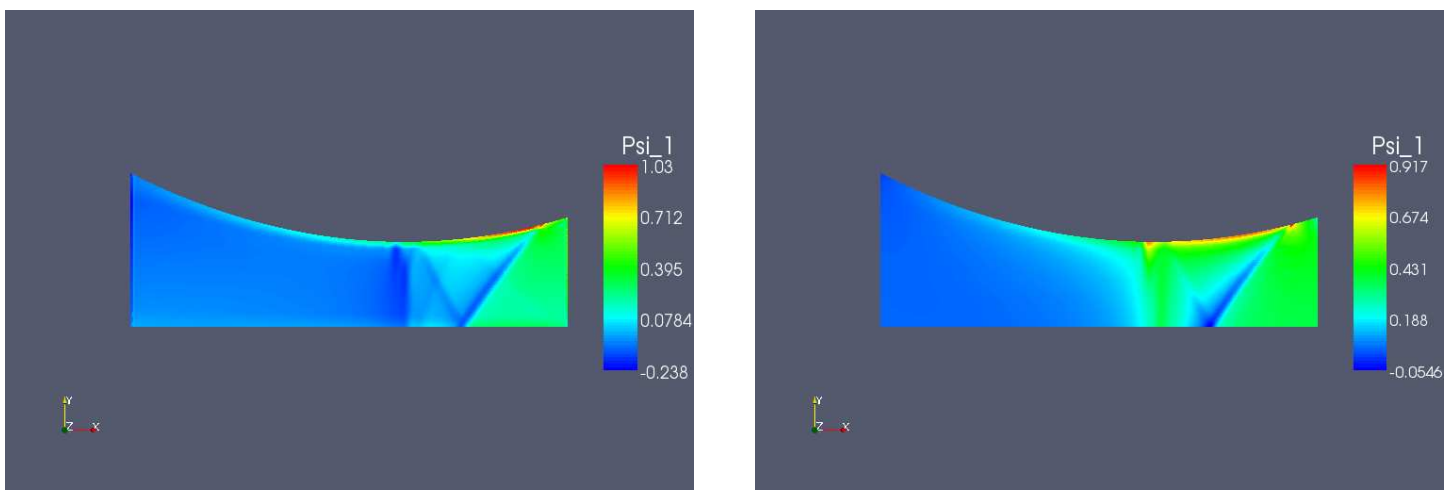

Figure 6. $\psi_{1}$ adjoint solutions comparison. Left, homogeneous boundary conditions. Right, characteristics-based boundary conditions.

A series of validation tests was devised to verify our approach. The results show good agreement with the conventional, widely accepted, treatment of the adjoint boundary problem, as it is reported in the literature for external flows. Nonetheless, there are small, yet apparently significant, differences between the two. Further tests are required to ascertain the influence the position of the far-field boundary exerts on the overall solution.

As for internal flows, test results have shown the full-characteristics conditions to yield smooth solutions in the neighborhood of permeable boundaries. As opposed to the sharp jumps that result when homogenous conditions are imposed there. That piece of evidence, along with an analogy with the flow physics, seem to indicate that the latter treatment of the boundary problem is inappropriate. However, it must be noted that we have not run full inverse design tests in that particular case.

\section{Acknowledgments}

The authors acknowledge the contribution of the NDF laboratory at the University of São Paulo in providing the required resources for the execution of this work. The first author also acknowledges the Conselho Nacional de Desenvolvimento Científico e Tecnológico (CNPq) for his Master's scholarship.

\section{References}

${ }^{1}$ Jameson, A., "Aerodynamic Design Via Control Theory," 12th IMACS World Congress on Scientific Computation, MAE Report 1824, Paris, July 1988.

${ }^{2}$ Hicks, R. M., Murman, E. M., and Vanderplaats, G. N., "An Assessment of Airfoil Design by Numerical Optimization," Report, Ames Research Center, Moffett Field, CA, July 1974, NASA TM X-3092.

${ }^{3}$ Hicks, R. M. and Henne, P. A., "Wing Design by Numerical Optimization," Journal of Aircraft, Vol. 15, No. 7, July 1978, pp. 407-412.

${ }^{4}$ Santos, L. C. C., A Hybrid Inverse Optimization Method for Aerodynamic Design of Lifting Surfaces, Ph.D. thesis, Georgia Institute of Technology, 1993.

${ }^{5}$ Pirroneau, O., "On Optimal Profiles in Stokes Flow," Journal of Fluid Mechanics, Vol. 59, No. 1, 1973, pp. 117-128.

${ }^{6}$ Pirroneau, O., "On Optimal Design in Fluid Dynamics," Journal of Fluid Mechanics, Vol. 64, No. 1, 1974, pp. 97-110.

${ }^{7}$ Pirroneau, O., Optimal Shape Design for Elliptic Systems, Springer-Verlag, 1st ed., 1983.

8 Jameson, A., "Aerodynamic Design Via Control Theory," Journal of Scientific Computing, Vol. 3, 1988, pp. 233-260.

${ }^{9}$ Cabuk, H., Sung, C.-H., and Modi, V., "Adjoint Operator Approach to Shape Design for Incompressible Flows," 3rd International Conference on Inverse Design Concepts and Optimization in Engineering Sciences (ICIDES), College Park, PA, 1991, pp. 391-404.

${ }^{10}$ Taasan, S., Kuruvila, G., and Salas, M. D., "Aerodynamic Design and Optimization in One Shot," 30th Aerospace Sciences Meeting and Exhibit, Reno, NV, January 1992, AIAA 92-0025.

${ }^{11}$ Kuruvila, G., Taasan, S., and Salas, M. D., "Airfoil Optimization by the One-Shot Method, Optimum Design Methods in Aerodynamics," AGARD-FDP-VKI Special Course, 1994.

12 Jameson, A., "Optimum Aerodynamic Design using CFD and Control Theory," AIAA 95-1729-CP, 1995.

${ }^{13}$ Santos, L. C. C., "A Study on Aerodynamic Design Optimization Using An Adjoint Method," Tech. Rep. IB 129 - 95/12, Institut für Entwurfsaerodynamik, Braunschweig, July 1995.

${ }^{14}$ Jameson, A., Martinelli, L., and Pierce, N. A., "Optimum Aerodynamic Design Using the Navier-Stokes Equations," 35th Aerospace Sciences Meeting \& Exhibit, American Institute of Aeronautics and Astronautics, AIAA, Reno, NV, January 1997, pp. $1-20$. 
Table 1. Coefficients

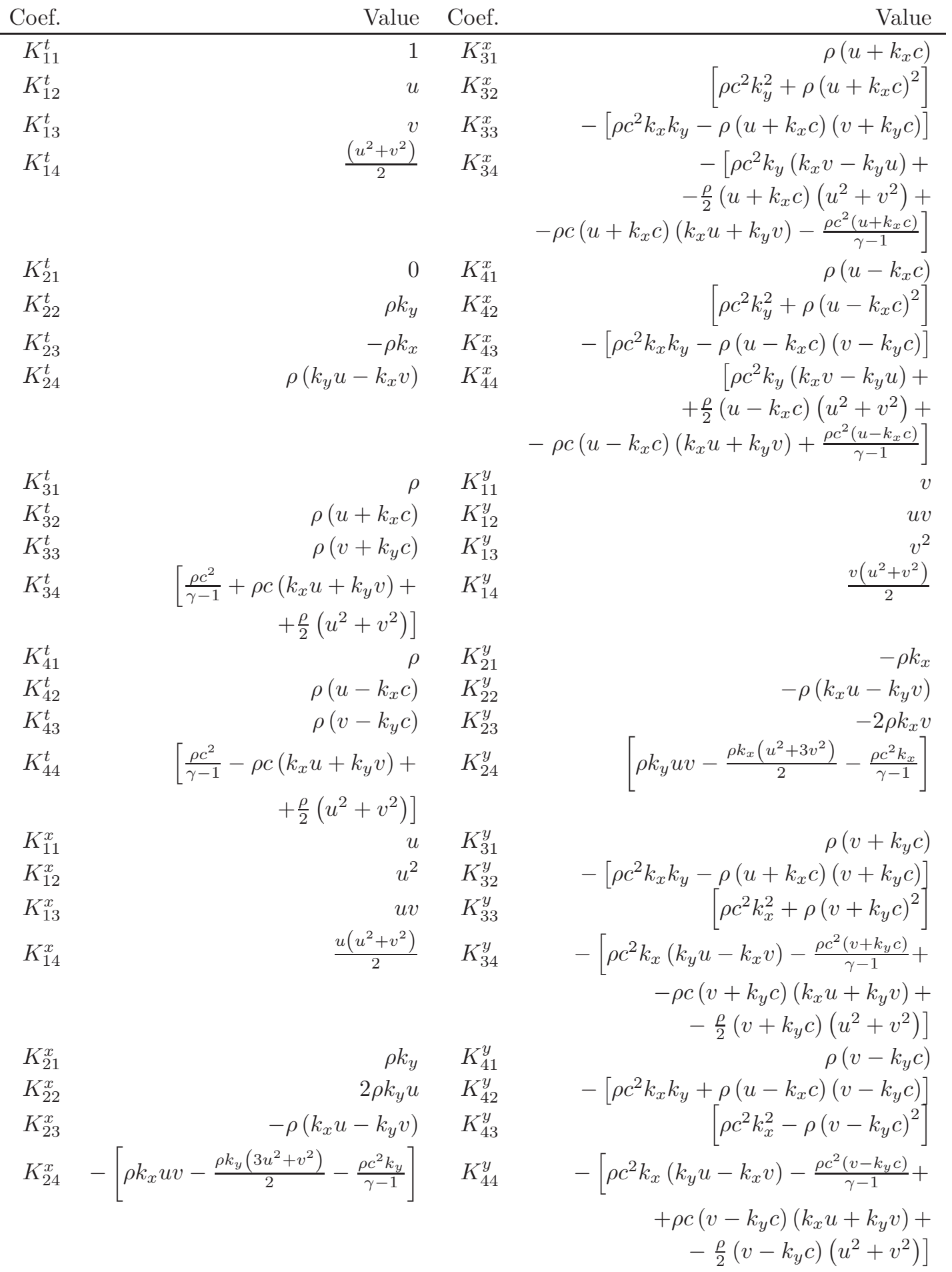


${ }^{15} \mathrm{Kim}$, S., Design Optimization of High-Lift Configurations Using a Viscous Adjoint-Based Method, Ph.D. thesis, Stanford University, 2001.

${ }^{16}$ Mohammadi, B. and Pirroneau, O., Applied Shape Optimization for Fluids, Oxford University Press, 1st ed., 2001.

${ }^{17}$ Alonso, J. J. and Kroo, I. M., "Advanced Algorithms for Design and Optimization of Quiet Supersonic Platforms," AIAA Computational Fluid Dynamics Conference, Reno, NV, January 2002, AIAA-2002-0144.

${ }^{18}$ Cacuci, D. G., Weber, F., C., M., O. E., and Marable, J. H., "Sensitivity Theory for General Systems of Non-Linear Equations," Nuclear Science and Engineering, Vol. 75, 1980, pp. 88-110.

${ }^{19}$ Hall, M. and Cacuci, D., "Physical Interpretation of the Adjoint Functions for Sensitivity Analysis of Atmospheric Models," Journal of Atmospheric Sciences, Vol. 40, October 1983, pp. 2537-2546.

${ }^{20}$ Nadarajah, S. K., The Discrete Adjoint Approach to Aerodynamic Shape Optimization, Ph.D. thesis, Stanford University, 2003.

${ }^{21}$ Kim, H. and Nakahashi, K., "Unstructured Adjoint Method for Navier-Stokes Equations," JSME International Journal, Vol. 48, No. 2, 2005

${ }^{22}$ Kim, S., Alonso, J. J., and Jameson, A., "Multi-Element High-Lift Configuration Design Optimization Using Viscous Continuous Adjoint Method," Journal of Aircraft, Vol. 41, No. 5, September-October 2004.

${ }^{23}$ Kim, H. J., Sasaki, D., Obayashi, S., and Nakahashi, K., "Aerodynamic Optimization of Supersonic Transport Wing Using Unstructured Adjoint Method," AIAA Journal, Vol. 39, No. 6, June 2001, pp. 1011-1020.

${ }^{24}$ Qiao, Z., Qin, X., and Yang, X., "Wing Design by Solving Adjoint Equations," 40th Aerospace Sciences Meeting 8 Exhibit, American Institute of Aeronautics and Astronautics, AIAA, Reno, NV, January 2002.

${ }^{25}$ Duta, M. C., Giles, M. B., and Campobasso, M. S., "The harmonic adjoint approach to unsteady turbomachinery design," Int. J. Numer. Meth. Fluids, Vol. 40, 2002, pp. 323-332.

${ }^{26}$ Thomas, J. P., Hall, K. C., and Dowell, E. H., "Discrete Adjoint Approach for Modeling Unsteady Aerodynamic Design Sensitivities," AIAA Journal, Vol. 43, No. 9, September 2005, pp. 1931-1936.

${ }^{27}$ Nadarajah, S. and Jameson, A., "Optimum Shape Design for Unsteady Three-Dimensional Viscous Flows using a NonLinear Frequency Domain Method," 24th Applied Aerodynamics Conference, American Institute of Aeronautics and Astronautics, AIAA, San Francisco, CA, June 2006.

${ }^{28}$ Nadarajah, S. K. and Jameson, A., "Optimum Shape Design for Unsteady Flows with Time-Accurate Continuous and Discrete Adjoint Methods," AIAA Journal, Vol. 45, No. 7, July 2007, pp. 1478-1491.

${ }^{29}$ Mani, K. and Mavriplis, D., "Unsteady Discrete Adjoint Formulation for Two-Dimensional Flow Problems with Deforming Meshes," AIAA Journal, Vol. 46, No. 6, June 2008, pp. 1351-1364.

${ }^{30}$ Giles, M., Pierce, N., and Süli, E., "Progress in adjoint error correction for integral functionals," Comput Visual Sci, Vol. 6, 2004, pp. 113-121.

${ }^{31}$ Giles, M. and Süli, E., "Adjoint methods for PDEs: a posteriori error analysis and postprocessing by duality," Acta Numerica, Vol. 11, 2002, pp. 145-236.

${ }^{32}$ Venditti, D. A. and Darmofal, D. L., "A multilevel error estimation and grid adaptive strategy for improving the accuracy of integral outputs," AIAA Paper 99-3292, 1999.

${ }^{33}$ Venditti, D. A. and Darmofal, D. L., "Adjoint Error Estimation and Grid Adaptation for Functional Outputs: Application to Quasi-One-Dimensional Flow," Journal of Computational Physics, Vol. 164, 2000, pp. 204-227.

${ }^{34}$ Venditti, D. A. and Darmofal, D. L., "Anisotropic grid adaptation for functional outputs: application to two-dimensional viscous flows," Journal of Computational Physics, Vol. 187, 2003, pp. 22-46.

${ }^{35}$ Giles, M. B. and Pierce, N. A., "Superconvergent lift estimates through adjoint error analysis," 1998, http://citeseerx.ist.psu.edu/viewdoc/summary?doi=10.1.1.27.152.

${ }^{36}$ Giles, M. B. and Pierce, N. A., "Adjoint recovery of superconvergent functionals from approximate solutions of partial differential equations," Report 98/18, Oxford University Computing Laboratory, Oxford, August 1999.

${ }^{37}$ Giles, M. B. and Pierce, N. A., "Improved lift and drag estimates using adjoint Euler equations," AIAA Paper 99-3293, 1999.

${ }^{38}$ Pierce, N. and Giles, M., "Adjoint and defect error bounding and correction for functional estimates," Journal of Computational Physics, Vol. 200, 2004, pp. 769-794.

${ }^{39}$ Jameson, A., Martinelli, L., and Pierce, N. A., "Optimum Aerodynamic Design Using the Navier-Stokes Equations," Theoretical and Computational Fluid Dynamics, Vol. 1, No. 10, 1998, pp. 213-237.

${ }^{40}$ Jameson, A., Sriram, A., and Martinelli, L., "A Continuous Adjoint Method for Unstructured Grids," AIAA Computational Fluid Dynamics Conference, Orlando, FL, June 2003, AIAA 2003-3955.

${ }^{41}$ Jameson, A. and Nadarajah, S. K., "A Comparison of The Continuous and Discrete Adjoint Approach to Automatic Aerodynamic Optimization," AIAA 38th Aerospace Sciences Meeting and Exhibit, Reno, NV, January 2000, AIAA $2000-0667$.

${ }^{42}$ S. Kim, J. J. A. and Jameson, A., "A Gradient Accuracy Study for the Adjoint-Based Navier-Stokes Design Method," AIAA Computational Fluid Dynamics Conference, 1999, AIAA-99-0299.

${ }^{43}$ Giles, M. B. and Pierce, N. A., "Adjoint Equations in CFD: Duality, Boundary Conditions and Solution Behavior," AIAA Paper 97-1850, 1997.

${ }^{44}$ Giles, M. B. and Pierce, N. A., "On the Properties of Solutions of the Adjoint Euler Equations," Numerical Methods for Fluid Dynamics VI. ICFD, edited by M. J. Baines, June 1998.

${ }^{45}$ Giles, M. B. and Pierce, N. A., "An introduction to the adjoint approach to design," Flow, Turbulence and Control, Vol. 65, No. 3, March 2000, pp. 393-415.

${ }^{46}$ Giles, M. B. and Pierce, N. A., "Analytic Adjoint Solutions for the Quasi-1D Euler Equations," Journal of Fluid Mechanics, Vol. 426, 2001, pp. 327-345.

${ }^{47}$ Volpe, E. V. and Santos, L. C. C., "Boundary and Internal Conditions for Adjoint Fluid Flow Problems - Applications to Quasi-1D Euler Equations," Journal of Engineering Mathematics, Vol. 65, No. 1, September 2009, pp. 1-24. 
${ }^{48}$ Gelfand, I. M. and Fomin, S. V., Calculus of Variations, Selected Russian Publications in Mathematical Sciences, Prentice-Hall, Inc., N.J., 1st ed., 1963, Translated and Edited by R. A. Silverman.

${ }^{49}$ Reuther, J. J., Aerodynamic Shape Optimization Using Control Theory, Ph.D. thesis, University of California, 1996.

${ }^{50}$ Jameson, A. and Kim, S., "Reduction of the Adjoint Gradient Formula for Aerodynamic Shape Optimization Problems," AIAA Journal, Vol. 41, No. 11, November 2003, pp. 2114-2129.

${ }^{51}$ Hayashi, M. T., Estudo Conceitual do Problema Adjunto baseado nas Equações de Euler para Aplicações de Otimização Aerodinâmica, Master's thesis, Escola Politécnica da Universidade de São Paulo, Fevereiro 2009.

${ }^{52}$ Hirsch, C., Numerical Computation of Internal and External Flows, Vol. II of Wiley Series in Numerical Methods in Engineering, John Wiley \& Sons, NY, 1st ed., 1994, Computational Methods for Inviscid and Viscous Flows.

${ }^{53}$ Hirsch, C., Numerical Computation of Internal and External Flows, Vol. I of Wiley Series in Numerical Methods in Engineering, John Wiley \& Sons, NY, 1st ed., 1994, Fundamentals of Numerical Discretization.

${ }^{54}$ Flügge, W., Tensor Analysis and Continuum Mechanics, Springer-Verlag, N.Y., 1st ed., 1972.

${ }^{55}$ Kaplan, W., Advanced Calculus, Addison-Wesley Pub. Co., N.Y., 1971.

${ }^{56}$ Ceze, M. A. B., Projeto Inverso Aerodinâmico Utilizando o Método Adjunto Aplicado às Equações de Euler, Master's thesis, Escola Politécnica da Universidade de São Paulo, Agosto 2008.

${ }^{57}$ Kulfan, B. M. and Bussoletti, J. E., "Fundamental Parametric Geometry Representations for Aircraft Component Shapes," 11th AIAA/ISSMO Multidisciplinary Analysis and Optimization Conference, Portsmouth, VA, September 2006.

${ }^{58}$ Ceze, M. A. B., Hayashi, M. T., and Volpe, E. V., "A Study of the CST Parameterization Characteristics," AIAA Applied Aerodynamics Conference, AIAA, San Antonio, TX, June 2009, AIAA 2009-3767.

${ }^{59}$ Jameson, A., Schimidt, W., and Turkel, E., "Numerical Solution of the Euler Equations by Finite Volume Methods Using Runge-Kutta Time-Stepping Schemes," AIAA Journal, 1981. 